\title{
Particle size distribution of Smoluchowski coagulation equation for Brownian motion at equilibrium
}

Xie Mingliang

State Key Laboratory of Coal Combustion, Huazhong University of Science and Technology, Wuhan 430074, China

Email: mlxie@mail.hust.edu.cn

Abstract: The information entropy for Smoluchowski coagulation equation is proposed based on statistical mechanics. And the normalized particle size distribution is a lognormal function at equilibrium from the principle of maximum entropy and moment constraint. The geometric mean volume and standard deviation in the distribution function are determined as simple constant. The results reveal that the assumption that algebraic mean volume be unit in self-preserving hypothesis is reasonable in some sense. Based on the present definition of information entropy, the Cercignani's conjecture holds naturally for Smoluchowski coagulation equation. Together with the proof that the conjecture is also true for Boltzmann equation, Cercignani's conjecture will holds for any two-body collision systems, which will benefit the understanding of Brownian motion and molecule kinematic theory, such as the stability of the dissipative system, and the mathematical theory of convergence to thermodynamic equilibrium.

Keywords: Particle size distribution, population balance equation, information entropy, moment method

\section{Introduction}

Population balance equation (PBE) are general mathematical framework for modeling of particulate system. In the framework of mono-variants internal coordinate and time for each particle, the PBE characterized as Smoluchowski coagulation equation (SCE), which takes the form [1]:

$$
\frac{\partial n(v, t)}{\partial t}=\frac{1}{2} \int_{0}^{v} \beta\left(v, v-v_{1}\right) n\left(v_{1}, t\right) n\left(v-v_{1}, t\right) d v_{1}-\int_{0}^{\infty} \beta\left(v_{1}, v\right) n(v, t) n\left(v_{1}, t\right) d v_{1}
$$

in which $n(v, t) d v$ is the number of particles per unit spatial volume with particle volume from $\mathrm{v}$ to $\mathrm{v}+\mathrm{dv}$ at time $\mathrm{t}$; and $\beta$ is the collision frequency function of coagulation. In the free molecule regime, the collision frequency function for Brownian coagulation is

$$
\beta=B_{1}\left(1 / v+1 / v_{1}\right)^{1 / 2}\left(v^{1 / 3}+v_{1}^{1 / 3}\right)^{2}
$$

where the constant $B_{1}=(3 / 4 \pi)^{1 / 6}\left(6 k_{B} T / \rho_{p}\right)^{1 / 2}, k_{B}$ is the Boltzmann constant; $\mathrm{T}$ is the temperature; and $\rho_{p}$ is the particle density. In the continuum regime, the corresponding collision frequency function is

$$
\beta=B_{2}\left(1 / v^{1 / 3}+1 / v_{1}^{1 / 3}\right)\left(v^{1 / 3}+v_{1}^{1 / 3}\right)
$$


where the constant $B_{2}=3 k_{B} T / 2 \mu$, and $\mu$ is the gas viscosity.

For the nonlinear partial integrodifferential structure of SCE, only a limited number of known analytical solution exist for simple coagulation kernel [2-5], If the collision frequency function is a homogeneous function of its arguments, the SCE can be converted into an ordinary integrodifferential equation by a similarity transformation [6]. The previous studies indicate that the particle size distribution (PSD) of an aged coagulating system approaches a universal asymptotic form called the self-preserving PSD [7-11]. If the self-preserving hypothesis can be firmly established, the tedious job of the determination of PSD can be reduced to the determination of only a few parameters for aged system [12-16]. Although the self-preserving hypothesis has been verified by some experiments and numerical methods [17-19], it is unfortunately that the algebraic mean volume of PSD is assumed to be unit in the derivation. This assumption turns the similarity transformation into a simple separated variable method, which makes the self-preserving hypothesis subject to great constraints in theoretically.

Because of the relative simplicity of implementation and low computational costs, the moment method has become a powerful tool for investigating the SCE [20-24]. Recently, Yu et al. [25] proposed a moment-based approach called the Taylor-series expansion method of moment (TEMOM) to analyze the evolution of particle number density function due to Brownian motion. The main idea of the TEMOM is that the nonlinear collision kernel is approximated by a Taylor-series polynomial, and the higher and fractional order moments based on PSD are approximated by the first three integer order moments, and the original TEMOM model achieves self-closure. In addition, the derivation of the TEMOM is completely based on a mathematical method, and no artificial assumption is introduced. The estimated truncation error of TEMOM model for Brownian coagulation has been examined [26], and the particle dimensionless moments corresponding to the deviation of PSD tends to a constant at long time [27], which is consistent with the self-preserving hypothesis. This method provides a new way for analyzing the coagulation problem theoretically. The moment method can only get finite moments, but the PSD is equivalence with the infinite moments. How to get the PSD from the finite moments is still a hot issue and challenge in science and engineering [28]. In addition, the expansion point is usually selected as the algebraic mean volume, whose value increases with time according to the asymptotic solution [27]. Whether there is an equilibrium state cannot be determined by the TEMOM itself.

According to the rule of statistical physics, Shannon information entropy is interpreted as a state function of a thermodynamic system and is proportional to the total number of particles [29]. The reduction in the total number of particles corresponds to a reduction in the information entropy of a particle system, which must be accompanied by a change of system energy in a dissipative system in accordance with the second law of thermodynamics. Brownian coagulation can be considered as a perfectly inelastic collision process, in which the colliding particles stick together and the maximum amount of kinetic energy of the system is lost. Then the thermodynamic constraint of Brownian coagulation can be proposed based on the binary perfectly inelastic collision theory and principle of maximum entropy [30, 31], which can be regarded as an adjoint equation of the SCE. The thermodynamic constraint can be used 
to explain the meaning of Eotvos constant in statistical mechanics [32], and its value can be quantitatively calculated by the equality at equilibrium. The constraint also gives the expression of the critical time to reach the thermodynamic equilibrium, which can be used to determine whether the PSD reaches the self-preserving form. In addition, the critical time is proportional to temperature, which can explain the long-time existence of urban fine particulate matter in northern China [33], and the structure of macroscopic agglomerates [34]. However, many other physical quantities are introduced into the thermodynamic analysis, such as specific surface free energy, internal energy and chemical potential, etc. These physical quantities are not strongly related to the SCE itself, and makes the analysis more complicated and quasi-empirical.

In this study, the definition of information entropy is proposed based on the SCE itself. According to the principle of maximum entropy and moment method, it is tried to give the PSD of SCE at equilibrium, and determine the expression for the algebraic mean volume and standard deviation of PSD.

\section{Moment method and its asymptotic solution}

In the moment method, the $\mathrm{k}$-th order moment $M_{k}$ of PSD is defined as

$$
M_{k}=\int_{0}^{\infty} v^{k} n(v) d v
$$

Using the moment transformation, the SCE turns to a series of equations related to the evolution of particle moments:

$$
\frac{d M_{k}}{d t}=\frac{1}{2} \int_{0}^{\infty} \int_{0}^{\infty}\left[\left(v+v_{1}\right)^{k}-v^{k}-v_{1}^{k}\right] \beta\left(v, v_{1}\right) n(v, t) n\left(v_{1}, t\right) d v d v_{1}
$$

The minimum set of moments required to close the particle moment equation is the first three, $M_{0}, M_{1}$ and $M_{2}$. The zeroth order moment represents the total particle number concentration; the first order moment is proportional to the total particle mass concentration, which remains constant due to the rigorous mass conservation requirement; and the second order moment describes the dispersion of PSD. In the free molecular regime, the TEMOM model is

$$
\begin{aligned}
\frac{d M_{0}}{d t} & =\frac{\sqrt{2} B_{1}\left(65 M_{C}{ }^{2}-1210 M_{C}-9223\right) M_{0}^{2}}{5184} \frac{M_{1}^{1 / 6}}{M_{0}^{1 / 6}} \\
\frac{d M_{1}}{d t} & =0 \\
\frac{d M_{2}}{d t} & =-\frac{\sqrt{2} B_{1}\left(701 M_{C}^{2}-4210 M_{C}-6859\right) M_{1}^{2}}{2592} \frac{M_{1}^{1 / 6}}{M_{0}^{1 / 6}}
\end{aligned}
$$

In the continuum regime, the corresponding model is 


$$
\begin{aligned}
\frac{d M_{0}}{d t} & =\frac{B_{2}}{81}\left(2 M_{C}^{2}-13 M_{C}-151\right) M_{0}^{2} \\
\frac{d M_{1}}{d t} & =0 \\
\frac{d M_{2}}{d t} & =-\frac{2 B_{2}}{81}\left(2 M_{C}{ }^{2}-13 M_{C}-151\right) M_{1}^{2}
\end{aligned}
$$

where the dimensionless particle moment is defined as

$$
M_{C}=\frac{M_{0} M_{2}}{M_{1}^{2}}
$$

Which corresponds to the variance of distribution function. For the TEMOM model of Brownian coagulation, the scaling asymptotic growth rate can be found as [27]

$$
-\frac{1}{M_{0}} \frac{d M_{0}}{d t}=\frac{1}{M_{2}} \frac{d M_{2}}{d t}=\frac{C}{t}
$$

where the constant $\mathrm{C}=6 / 5$ in the free molecule regime, and $\mathrm{C}=1$ in the continuum regime, respectively. It can be found that the particle dimensionless moment tends to a constant with the asymptotic solution, i.e.,

$$
\frac{d M_{C}}{d t}=\frac{d}{d t}\left(\frac{M_{0} M_{2}}{M_{1}^{2}}\right)=0
$$

The asymptotic solution reveals that the zeroth order particle moment tends to zero as time advances. Is this solution the reality? This question cannot be answered by the moment method itself. The traditional way to solve this problem is to propose the constraint condition based on the second law of thermodynamics. However, this approach is quasi-empirical. Another way is to define the entropy of SCE itself, and the PSD at equilibrium is given according to the principle the maximum entropy. But how to give an appropriate definition of entropy is a problem in itself. There are two main definitions of entropy of SCE in the literatures, one is the definition of entropy based on the PSD, and the other is that based the coagulation probability. The latter seems to be more complex than the former, and can provide more information to get the best fractal dimension of agglomerates with simple collision kernel at equilibrium. In our previous work, the two kinds of definitions of entropy are equivalent with asymptotic analysis for Brownian coagulation, and both can only solve the problem partially. In this work, the entropy will be defined based on statistical mechanics, which overcomes the shortcomings of the previous definition of entropy and successfully determines the algebraic mean volume.

\section{Information entropy of SCE}

Smoluchowski coagulation equation is deferent from the Boltzmann equation [35]. In the former, the collision between particles is perfectly inelastic, the mass and 
momentum are conserved while the maximum amount of kinetic energy of system is lost, and the total particle number density is reduced. Therefore, Smoluchowski coagulation equation is a soft spheres collision model. However, the Boltzmann equation is a hard spheres collision model, the conservation of mass and momentum together with the conservation of kinetic energy makes possible the calculation of final velocities in two-body collision, and the particle number density remains constant. Therefore, the definition of information entropy for SCE is also deferent from that for Boltzmann equation. In the latter, the information entropy is the only the function of the integral of the product of the distribution function and its logarithm.

In the literatures, there are several definitions of information entropy for SCE, such as the definition based on the coagulation probability [36], but the effect of particle number density on the entropy is not considered. Another way to define the entropy for SCE based on thermodynamics can be found in our previous work [30,31], the properties and evolution of entropy for SCE are studied from the perspective of energy balance and conversion, not from the SCE itself.

Starting from the original definition of information entropy [29], which is a function of the total number of microscopic states $(\Omega)$ in a disperse system, i.e., $\mathrm{S}=k_{B} \ln \Omega$, and $\Omega$ can be calculated for SCE as

$$
\Omega=\frac{M_{0} !}{\sum n !}
$$

According to the Sterling formula, then the information entropy can be expressed as

$$
S=k_{B}\left(M_{0} \ln M_{0}-M_{0}\right)-k_{B} \sum(n \ln n-n)
$$

Therefore, the information entropy for SCE can be considered as a function of PSD and the total number of particles. Let the normalized PSD as

$$
p(v, t)=\frac{n(v, t)}{\int_{0}^{\infty} n(v, t) d v}=\frac{n(v, t)}{M_{0}}
$$

Then the information entropy can be simplified as form of continuity function

$$
S=-k_{B} M_{0} \int_{0}^{\infty} p(v, t) \ln p(v, t) d v
$$

\section{Principle of maximum entropy}

In mathematics, the principle of maximum entropy can be expressed as that the normalized PSD makes the information entropy approach to the extremum, i.e.,

$$
\max \left[S\left(M_{0}, p\right)=-k_{B} M_{0} \int_{0}^{\infty} p(v, t) \ln p(v, t) d v\right]
$$

with the moment constraints as 


$$
\begin{aligned}
& \int_{0}^{\infty} p(v, t) d v=1, \\
& \int_{0}^{\infty} v p(v, t) d v=\frac{M_{1}}{M_{0}}, \\
& \int_{0}^{\infty} v^{2} p(v, t) d v=\frac{M_{2}}{M_{0}},
\end{aligned}
$$

Based on the variational principle and Lagrange multiplier method, the normalized PSD under the condition of maximum entropy can be obtained as a lognormal function:

$$
p(v, t)=\frac{1}{3 \sqrt{2 \pi} \ln \sigma} \exp \left[-\frac{\ln ^{2}\left(v / v_{g}\right)}{18 \ln ^{2} \sigma}\right] \frac{1}{v}
$$

In which the geometric mean volume $\mathrm{vg}_{\mathrm{g}}$ and standard deviation $\sigma$ can be expressed as the functions of particle moments

$$
v_{g}=\frac{M_{1}^{2}}{M_{0}^{3 / 2} M_{2}^{1 / 2}}, \ln ^{2} \sigma=\frac{1}{9} \ln M_{C}
$$

Then the information entropy at equilibrium can be expressed as

$$
S^{e q}=M_{0}\left[\frac{1}{2}+\frac{1}{2} \ln \left(\frac{2 \pi \ln M_{C}}{M_{C}}\right)+\ln \left(\frac{M_{1}}{M_{0}}\right)\right]
$$

Which are the functions of particle dimensionless moment $M_{\mathrm{C}}$ and zeroth order moment $M_{0}$. Using the extreme condition again, i.e.,

$$
\frac{\partial S^{e q}}{\partial M_{C}}=0, \frac{\partial S^{e q}}{\partial M_{0}}=0
$$

The parameters of PSD can be determined as

$$
M_{C}^{e q}=e, M_{0}^{e q}=M_{1} \sqrt{2 \pi} / e
$$

Or

$$
\sigma^{e q}=\sqrt[3]{e}, v_{g}^{e q}=\sqrt{e / 2 \pi}
$$

And the information entropy at equilibrium is simplified as

$$
S^{e q}=k_{B} M_{0}^{e q}
$$

And algebraic mean volume at equilibrium is 


$$
\left(\frac{M_{1}}{M_{0}}\right)^{e q}=\frac{e}{\sqrt{2 \pi}} \sim 1.0844
$$

The result reveals that the assumption that $\left(M_{1} / M_{0}\right)^{\mathrm{eq}}=1$ in the self-preserving hypothesis is reasonable in some sense.

\section{Cercignani's conjecture for SCE}

Cercignani's conjecture is based on the entropy-entropy production method, which was first used in kinetic theory for the Fokker-Planck equation [37], it has been at the core of the renewal of the mathematical theory of convergence to thermodynamically equilibrium for Boltzmann equation over the past decade [38]. In explicitly, Cercignani's conjecture assumes a linear inequality between the entropy and the entropy production functional for Boltzmann integral operator as

$$
\frac{d S}{d t} \geq K\left(S^{e q}-S\right)
$$

In which $\mathrm{K}$ is the proportional coefficient. Recently, Cercignani's conjecture for Boltzmann equation with hard sphere collision has been proved by Villani [39]. Brownian coagulation is a similar dissipative system as that described by Boltzmann equation. Is this conjecture still true for the SCE?

In 2019, Xie and Liu [40] have proposed a similar equality of the relationship between entropy and entropy production for SCE as the structure of Cercignani's conjecture according to the thermodynamic constraint. In their derivation process, a similar $\mathrm{H}$ function as that in Boltzmann equation is defined, but the relationship between the new $\mathrm{H}$ function and entropy $\mathrm{S}$ remains unknown. Because the entropy in that work is based on thermodynamics, it is difficult to answer this question theoretically. Can the definition of information entropy in present work solve this problem?

The entropy production can be calculated from the definition of entropy in Eq.(12) as

$$
\frac{d S}{d t}=-\frac{1}{M_{0}} \frac{d M_{0}}{d t}\left[-M_{0}^{2} \frac{d}{d M_{0}}\left(\frac{S}{M_{0}}\right)-S\right]
$$

According to the principle of maximum entropy, the entropy is less than that at equilibrium, i.e., $\mathrm{S} \leq \mathrm{S}^{\mathrm{eq}}$. In this way, the following inequality will hold,

$$
\frac{d S}{d t} \geq-\frac{1}{M_{0}} \frac{d M_{0}}{d t}\left[-M_{0}^{2} \frac{d}{d M_{0}}\left(\frac{S^{e q}}{M_{0}}\right)-S\right]=K\left(S^{e q}-S\right)
$$

In which the constant $\mathrm{K}$ is obtained

$$
K=-\frac{1}{M_{0}} \frac{d M_{0}}{d t}
$$


And the scaling growth rate of entropy can be obtained as

$$
\frac{1}{S} \frac{d S}{d t} \geq-\frac{1}{M_{0}} \frac{d M_{0}}{d t}\left[-\frac{M_{0}^{2}}{S} \frac{d}{d M_{0}}\left(\frac{S^{e q}}{M_{0}}\right)-1\right]=\frac{1}{M_{0}} \frac{d M_{0}}{d t}
$$

Therefore, the Cercignani's conjecture is true for SCE due to Brownian motion, which means that the effect of molecular collisions is to force a non-equilibrium distribution function at a point in physical space back to a lognormal distribution function, and SCE for Brownian motion is convergence in mathematically.

\section{Discussions and conclusions}

In this study, the information entropy for SCE is proposed from the point of view of total number of microscopic states in a disperse systems. Based on the variational principle and Lagrange multiplier method, the normalized PSD can be obtained as a lognormal function under the condition of maximum entropy and moment constraints. The information entropy at equilibrium are the functions of particle dimensionless moment and zeroth order moment. Using the extreme condition, the geometric mean volume and standard deviation are both determined as simple constants. The results are consistent with the self-preserving hypothesis. It should be pointed out that the geometric mean volume has not been determined in the previous literatures.

With the present definition of information entropy, the Cercignani's conjecture for SCE holds naturally. The result reveals that the SCE for Brownian motion is convergence in mathematically. As mentioned above, Cercignani's conjecture are both true for elastic and perfectly inelastic collision theory. Moreover, a general two-body collision can be decomposed into the weighted sum of elastic and perfectly inelastic collision. Therefore, Cercignani's conjecture holds for any two-body collision systems, which will explain many unknown problems. For example, the Navier-Stokes equation can be derived from the Boltzmann equation using Chapman-Enskog asymptotic expansion method, meanwhile, there is a dissipation of turbulent kinetic energy in turbulence flow. The question is that how can the dissipation of turbulent kinetic energy be realized in elastic collision system? Perhaps the combination of elastic and perfectly inelastic collision theory can solve this theoretical inconsistent problem.

\section{References}

1. Friedlander, S.K., 2000. Smoke, Dust, and Haze: Fundamentals of Aerosol Dynamics, 2nd edition Oxford University Press, London.

2. White, W.H., 1980. A global existence theorem for Smoluchowski's coagulation equation, Proc. Am. Math. Soc., 80, 273-276

3. Leyvraz, F., 2003. Scaling theory and exactly solved models in the kinetics of irreversible aggregation. Physics Reports, 383, 95-212

4. Blum, J., 2006. Dust agglomeration. Advances in Physics, 55, 881-947

5. Niethammer, B., Nota, A., Throm, S., Velazquez, J.J.L., 2019. Self-similar asymptotic behavior for the solution of a linear coagulation equation. Journal of Differential Equations, 266, 653-715 
6. Friedlander, S.K., Wang, C.S., 1966. The self-preserving particle size distribution for coagulation by Brownian motion. Journal of Colloid and Interface Science, 22, 126-132

7. Vicsek, T., Family, F., 1984. Dynamic scaling for aggregation of clusters, Physical Review Letters, 52, 1669

8. Van Dongen, P.G.J., Ernst, M.H., 1985. Dynamical scaling in the kinetics of clustering. Physical Review Letters, 54, 1396

9. Van Dongen, P.G.J. Ernst, M.H., 1988. Scaling solutions of Smoluchowski's coagulation equation. Journal of Statistical Physics, 50, 295-329

10. Dekkers, P.J., Friedlander, S.K., 2002. The self-preserving size distribution theory I. Effect of the Knudsen number on aerosol agglomerate Growth. Journal of Colloid and Interface Science, 248, 295-305

11. Leyvraz, F., 2006. Scaling theory for gelling systems: Work in progress. Physica D, 222, 21-28

12. Pratsinis, S.E., 1988. Simultaneous nucleation, condensation, and coagulation in aerosol reactor. Journal of Colloid and Interface Science, 124, 416-427

13. Van Dongen, P.G.J., 1989. Upper critical dimension in irreversible aggregation, Physical Review Letters, 63, 1281

14. Park, S., Lee, K., Otto, E., Fissan, H., 1999. Log-normal size distribution theory of Brownian aerosol coagulation for the entire particle size range: Part I-analytical solution using the harmonic mean coagulation. Journal of Aerosol Science, 30, 316

15. Otto, E., Fissan, H., Park, S., Lee, K., 1999. Log-normal size distribution theory of Brownian aerosol coagulation for the entire particle size range: Part II-analytical solution using Dahneke's coagulation kernel. Journal of Aerosol Science, 30, 1734

16. Canizo, J.A., Throm, S., 2021. The scaling hypothesis for Smoluchowski coagulation equation with bounded perturbations of the constant kernel. Journal of Differential Equations, 270, 285-342

17. Vemury, S., Kusters, K.A., Pratsinis, S.E., 1994. Time-lag for attainment of selfpreserving particle size distribution by coagulation. Journal of Colloid and Interface Science, 165, 53-59

18. Vemury, S., Pratsinis, S.E., 1995. Self-preserving size distribution of agglomerates. Journal Aerosol Science, 26, 175-185

19. Dekkers, P.J., Tuinman, I.L., Marijnissen, J.C.M., Friedlander, S.K., Scarlett B., 2002. The self-preserving size distribution theory II. Comparison with experimental results for $\mathrm{Si}$ and $\mathrm{Si}_{3} \mathrm{~N}_{4}$ aerosols. Journal of Colloid and Interface Science, 248, 306-314

20. Wooldridge, M.S., 1998. Gas-phase combustion synthesis of particles. Progress in Energy and Combustion Science, 24, 63-87.

21. Aldous, D.J., 1999. Deterministic and stochastic models for coalescence (aggregation and coagulation): A review of the mean field theory for probabilists. Bernoulli, 5, 3-48

22. Rigopoulos, S., 2010. Population balance modelling of polydispersed particles in 
reactive flows. Progress in Energy and Combustion Science, 36, 412-443

23. Liao, Y.X., Lucas, D., 2010. A literature review on mechanisms and models for the coalescence process of fluid particles. Chemical Engineering Science, 65, 28512864

24. Roth, P., 2007. Particle synthesis in flames. Proceedings of the Combustion Institute, 31, 1773-1788

25. Yu, M.Z., Lin, J.Z., Chan, T.L., 2008. A new moment method for solving the coagulation equation for particles in Brownian motion. Aerosol Science and Technology, 42, 705-713

26. Xie, M.L., 2016. Error estimation of TEMOM for Brownian coagulation. Aerosol Science and Technology, 50, 919-925

27. Xie, M.L., Wang, L.P., 2013. Asymptotic solution of population balance equation based on TEMOM model. Chemical Engineering Science, 94, 79-83

28. John, V., Angelov, I., Oncul, A.A., Thevenin, D., 2007. Techniques for the reconstruction of a distribution from a finite number of its moments. Chemical Engineering Science, 62, 2890-2904

29. Jaynes, E.T., 1957. Information theory and statistical mechanics. Physical Review, $106,620-630$

30. Xie, M.L., 2017. On the growth rate of particle specific surface area for Brownian coagulation. Journal of Aerosol Science, 113, 36-39

31. Xie, M.L., Yu, M.Z., 2018. Thermodynamic analysis of Brownian coagulation based on moment method. International Journal of Heat and Mass Transfer, 122, 922-928

32. Palit, S., 1956, Thermodynamic interpretation of the Eotvos constant. Nature, 177, $1180-1180$

33. Zhang, R., Wang, G., Guo, S., Zamora, M.L., Ying, Q., Lin, Y., Wang, W., Hu, M., Wang, Y., 2015. Formation of urban fine particulate matter, Chemical Review, 115, 3803-3855

34. Blum, J., Schrapler, R., 2004. Structure and mechanical properties of high-porosity macroscopic agglomerates formed by random ballistic deposition. Physical Review Letters, 93, 115503

35. Cercignani, C., 1988. The Boltzmann equation and its applications. SpringerVerlag Press, New York

36. Gmachowski, L., 2001. A method of maximum entropy modeling the aggregation kinetics. Colloids and Surfaces A: Physicochemical and Engineering Aspects, 176, 151-159

37. Toscani, G., 1999. Entropy production and the rate of convergence to equilibrium for the Fokker-Planck equation. Quarterly Journal of Applied Mathematics, 57, 521-541

38. Desvillettes, L., Villani, C., 2005. On the trend to global equilibrium for spatially inhomogeneous kinetic system: The Boltzmann equation. Inventions Mathematicae, 159, 245-316

39. Villani, C., 2003. Cercignani's conjecture is sometimes true and always almost true. Communication in Mathematical Physics, 234, 455-490 
40. Xie, M.L., Liu H., 2019. Cercignani's conjecture is almost true for Smoluchowski equation. Journal of Aerosol Science, 128, 14-16

\section{Appendix: Lagrange multiplier method and variational principle}

When the total number of particles is fixed, the principle of maximum entropy can be expressed as

$$
\max \left[\operatorname{Entropy}(p)=-\int p(x) \ln p(x) d x\right]
$$

With the mathematical and physical constraint

$$
\begin{aligned}
& \int p(x) d x=1 ; \\
& \int x p(x) d x=\ln \mu ; \\
& \int(x-\mu)^{2} p(x) d x=(\ln \sigma)^{2}
\end{aligned}
$$

According to the Lagrange multiplier method, three parameters are introduced as

$$
\begin{aligned}
L(p, \alpha, \beta, \gamma)= & -\int p(x) \ln p(x) d x+\alpha\left(\int p(x) d x-1\right) \\
& +\beta\left(\int x p(x) d x-\ln \mu\right)+\gamma\left(\int(x-\ln \mu)^{2} p(x) d x-(\ln \sigma)^{2}\right)
\end{aligned}
$$

Using the extreme condition, it can be found the following relationship

$$
\frac{\partial L}{\partial p}=\ln p(x)+1-\alpha-\beta x-\gamma(x-\ln \mu)^{2}=0
$$

And the distribution function can be expressed as

$$
p(x)=\exp (\alpha-1) \exp \left[\beta x+\gamma(x-\ln \mu)^{2}\right]
$$

By rearrangement, it becomes

$$
p(x)=\exp \left[\alpha-1+\gamma(\ln \mu)^{2}-\gamma\left(\frac{(2 \gamma \ln \mu-\beta)}{2 \gamma}\right)^{2}\right] \exp \left[\gamma\left(x-\frac{2 \gamma \ln \mu-\beta}{2 \gamma}\right)^{2}\right]
$$

From the integrability condition, it can be found

$$
\gamma<0
$$

From the expectation condition, it can be found

$$
E(x)=\ln \mu-\frac{\beta}{2 \gamma}=\ln \mu \text { and } \beta=0
$$

From the constraint that the integral of distribution is unit, it can be found

$$
\exp (\alpha-1) \sqrt{-\frac{\pi}{\gamma}}=1
$$


From the constraint of variance, it can be found that

$$
\exp (\alpha-1)\left(\frac{1}{2} \sqrt{\frac{\pi}{-\gamma^{3}}}\right)=(\ln \sigma)^{2}
$$

Then the solution can be found

$$
\gamma=-\frac{1}{2(\ln \sigma)^{2}}
$$

And the distribution function is obtained as

$$
p(x)=\frac{1}{\sqrt{2 \pi} \ln \sigma} \exp \left[-\frac{(x-\ln \mu)^{2}}{2(\ln \sigma)^{2}}\right]
$$

Using the coordinate transformation

$$
x=\ln y
$$

And the distribution function becomes

$$
p(x) d x=p(y) \frac{1}{y} d y=\frac{1}{\sqrt{2 \pi} \ln \sigma} \exp \left[-\frac{\ln ^{2}(y / \mu)}{2 \ln ^{2} \sigma}\right] \frac{1}{y} d y
$$

With the relationship between volume and particle size,

$$
v=\frac{1}{6} \pi y^{3}
$$

And the distribution function with particle volume is obtained as

$$
p(v, t)=\frac{1}{3 \sqrt{2 \pi} \ln \sigma} \exp \left[-\frac{\ln ^{2}\left(v / v_{g}\right)}{18 \ln ^{2} \sigma}\right] \frac{1}{v}
$$

Which is the lognormal function listed in Eq.(16) of the paper. 